\title{
Asymmetric Heat Transfer in Liquid-Liquid Segmented Flow in Microchannels
}

\author{
Zhizhao Che ${ }^{\mathrm{a}, *}$, Teck Neng Wong ${ }^{\mathrm{a}}$, Nam-Trung Nguyen ${ }^{\mathrm{b}}$, Chun Yang ${ }^{\mathrm{a}}$ \\ ${ }^{a}$ School of Mechanical and Aerospace Engineering, Nanyang Technological University, 50 \\ Nanyang Avenue, 639798, Singapore \\ ${ }^{b}$ Queensland Micro- and Nanotechnology Centre, Griffith University, 170 Kessels Road, \\ Brisbane, QLD 4111, Australia
}

\begin{abstract}
Heat transfer in segmented flow in microchannels can be significantly enhanced by recirculating vortices, due to the presence of interfaces. The processes of heat transfer in segmented flow subjecting to asymmetric boundary conditions are studied. Two types of boundary conditions are considered, asymmetric constant surface temperature and asymmetric constant surface heat flux. The paths of heat flow and the effects of the thermal conductivity, the plug length, and the Peclet number are studied. The results show different features from those at symmetric boundary conditions. The heat transfer process at asymmetric boundary conditions is controlled by both thermal advection and diffusion at the mid-plane of the channel. The coupling effects between the adjacent plugs complicate the process by the heat transfer across plug-plug interfaces.
\end{abstract}

Keywords: Segmented flow, Heat transfer, Thermal boundary layer, Multiphase microfluidics

\footnotetext{
*Corresponding author. Tel.: +65-67905587 Fax: +65-67911859

Email address: chez0001@e.ntu.edu.sg (Zhizhao Che)
} 


\section{Introduction}

Heat generated by microprocessors with ultra large scale integration causes a problem to many modern electronic devices and thus hinder their development. Efficient cooling is crucial for maintaining their desired performance. If heat cannot be removed immediately, microprocessors will become unreliable or be permanently destroyed. High heat flux cooling is also required in applications such as high heat-load optical components, laser diode arrays, and X-ray medical devices. Different methods have been proposed to cope with the high heat flux in heat exchangers, such as spray cooling [1-3], jet impingement [4], heat pipes [5, 6], and microchannels [79]. Heat transfer in microchannels is enhanced by large heat transfer areas per unit volume. Another advantage of microchannels is the possibility to directly integrate the cooling components into microchips to achieve efficient cooling.

In comparison with single phase microchannel heat exchangers, multiphase microchannel heat exchangers have a much higher efficiency, thanks to the recirculating vortices in multiphase flow. Due to the presence of interfaces, the recirculating vortices can enhance the heat transfer by advecting heated fluid from the wall to the central region of the microchannel, and by supplying fresh fluid from the central region of the microchannel to the wall $[10,11]$. The flow dynamics [12-15], mass transfer $[16,17]$ in segmented flow has been widely studied experimentally $[12,18]$, numerically, and analytically.

The heat transfer enhancement of multiphase flow in microchannels has 
been proven experimentally by several research groups. Betz and Attinger [19] performed experiments on a polycarbonate heat sink consisting of an array of seven parallel microchannels, using water and air to form segmented flow. Sharon et al. [20] utilized a copper tube with $2 \mathrm{~mm}$ inner diameter as the flow channel, while using water and nitrogen as the two immiscible phases. The temperature was measured using several thermal couples along the tube. Lim et al. [21] used borosilicate circular glass tubes with inner diameters of 300 and $500 \mu \mathrm{m}$, and a thin layer of Indium Tin Oxide (ITO) coating on the tubes as the heat source.

To analyze the heat transfer process in plug flow, Muzychka et al. $[22,23]$ proposed a simplified model to consider the whole process as a Graetz problem with steady state heat transfer. Numerical simulations were performed to study multiphase heat and mass transport in microchannels. Some simulations were based on fixed frames of references [24-26], while others were based on frames of references following the droplets/plugs $[27,28]$, which could significantly reduce the simulation time.

Besides the traditional pressure-driven method, another strategy to actuate droplets is to use electrowetting on dielectric (EWOD), which manipulates individual droplets by applying a series of electrical potentials to an array of patterned electrodes $[29,30]$. This subfield of microfluidics is often termed digital microfluidics. Digitized heat transfer on the basis of EWOD was proposed by Mohseni and Baird [31, 32]. In comparison with pressuredriven flow in microchannels, the digital approach could avoid high pressure problems, which is encountered in pressure-driven flow in microchannels at high flow rates. 
Although many investigations of heat transfer in segmented flow have been reported, many of the observed phenomena remain unclear. The investigation is challenging because heat transfer in segmented flow in microchannels is a complex process and involves many parameters. In the previous studies, we studied the heat transfer process of gas-liquid plug flow subjecting to constant surface temperature in two dimensional (2D) microchannels [10], and the heat transfer process of gas-liquid plug flow subjecting to constant surface heat flux in cylindrical microchannels [11]. The results showed that heat transfer could be significantly enhanced by the recirculating vortices. Here we extended this method to study the heat transfer process of liquid-liquid segmented plug trains moving in microchannel subjecting to asymmetric boundary conditions, where a plug train refers to a series of $N$ consecutive different plugs which repeat periodically in microchannels. With asymmetric boundary conditions, one wall of the microchannel is adiabatic, and the other wall is maintained at a constant surface temperature or a constant surface heat flux, as shown in Figure 1. When the flow in microchannel heat sinks is in the flow regimes of plug trains, the heat transfer benefits from the recirculating vortices, and is further enhanced by the interaction of both phases. In addition, asymmetric heat transfer problems are often encountered when only one side of the heat sink is in contact with heat sources.

For most microchannels, the typical widths are about several tens to hundreds of micrometers. At this scale, the continuity of mass, momentum, and energy is still valid for the liquid in the microchannels [33, 34], and is used in this investigation. This paper is organized as follows. The numerical 
(a)

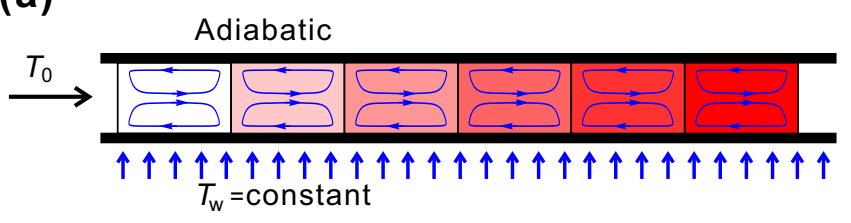

(b)

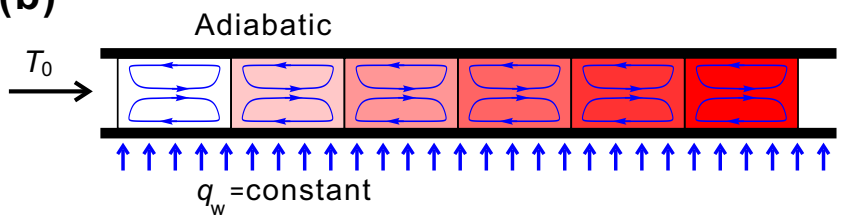

Figure 1: Schematic diagrams of asymmetric heat transfer in symmetric segmented flow in microchannels: (a) Asymmetric constant surface temperature boundary condition (abbreviated to TA): the bottom wall is maintained at a constant surface temperature while the top wall is adiabatic; (b) Asymmetric constant surface heat flux boundary condition (abbreviated to QA): the bottom wall is maintained at a constant surface heat flux while the top wall is adiabatic.

methods are described in Section 2. The results are presented and discussed in Section 3. The process of heat transfer is analyzed, the asymmetric features of heat transfer are discussed, and the effect of thermal conductivity is studied.

\section{Methods}

\subsection{Flow field in plug trains moving in microchannels}

To study the flow field in plug trains, a moving frame of reference was built on each plug following the plug train, as shown in Figure 2. The moving frames of reference were used for the flow field and the heat transfer. To reduce the computation time, a theoretical flow field for plug trains [35] was employed and substituted into the simulation of heat transfer process, 
which has the form of

$$
\begin{aligned}
\hat{u}_{x, i} & =\sum_{m=1}^{\infty}\left[p_{m}^{o^{\prime}}\left(\hat{y}_{i}\right) X_{m}^{o}+p_{m}^{e^{\prime}}\left(\hat{y}_{i}\right) X_{m}^{e}\right] \frac{(-1)^{m}}{\alpha_{m}} \cos \left(\alpha_{m} \hat{x}_{i}\right) \\
& +\hat{L}_{i} \sum_{l=1}^{\infty}(-1)^{l}\left[q_{l}^{e}\left(\hat{x}_{i}\right) Y_{l}^{e}+q_{l}^{o}\left(\hat{x}_{i}\right) Y_{l}^{o}\right] \cos \left(\beta_{l} \hat{y}_{i}\right) \\
\hat{u}_{y, i} & =\sum_{m=1}^{\infty}\left[p_{m}^{o}\left(\hat{y}_{i}\right) X_{m}^{o}+p_{m}^{e}\left(\hat{y}_{i}\right) X_{m}^{e}\right](-1)^{m} \sin \left(\alpha_{m} \hat{x}_{i}\right) \\
& -\hat{L}_{i} \sum_{l=1}^{\infty} \frac{(-1)^{l}}{\beta_{l}}\left[q_{l}^{e^{\prime}}\left(\hat{x}_{i}\right) Y_{l}^{e}+q_{l}^{o^{\prime}}\left(\hat{x}_{i}\right) Y_{l}^{o}\right] \sin \left(\beta_{l} \hat{y}_{i}\right)
\end{aligned}
$$

where $\hat{u}_{x} \equiv u_{x} / V, \hat{u}_{y} \equiv u_{y} / V, \hat{x} \equiv x / h, \hat{y} \equiv y / h, V$ is the speed of the plug train, $h \equiv w / 2$ is the half width of the microchannel, and $i$ is the index of the plug unit in the plug train. Simplification about the film has been made to theoretically study the plug train flow. It is valid when the liquid film is thin and its effect on the flow field and heat transfer is negligible. The thickness of the liquid film is mainly determined by the force balance between the viscous force and the surface tension force, which can be represented by the capillary number. At a low capillary number, the surface tension force is strong. The strong surface tension force can push the liquid away from the liquid film and results in a thin liquid film. As the capillary number increases, the effect of surface tension decreases while the effect of the viscous force increases. Then the thickness of the liquid film increases. A typical flow speed $0.1 \mathrm{~m} / \mathrm{s}$ of water corresponds to a capillary number of $1.3 \times 10^{-3}$, which leads to a film thickness of $1.5 \%$ of the radius of capillary tubes, based on the correlation in [36]. The details of the coefficients in Eqs.(1)-(2) and the method to obtain the theoretical solution are explained in [35]. In this paper, we only consider plug trains consisting of two plugs, as shown in Figure 2. Plug trains with 


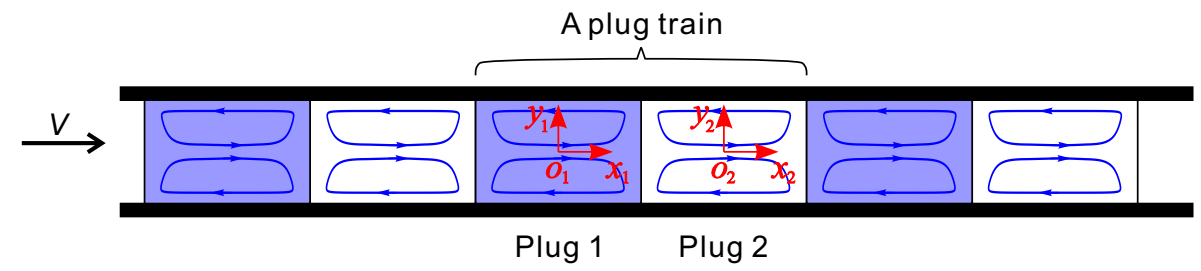

Figure 2: Schematic diagram of a typical plug train which consists of two immiscible liquid plugs of different fluid properties.

more plugs could be studied similarly.

\subsection{Simulation of heat transfer}

The governing equation for heat transfer in plug trains is expressed as

$$
\frac{\partial\left(\rho c_{p} T\right)}{\partial t}+\frac{\partial\left(\rho c_{p} u_{x} T\right)}{\partial x}+\frac{\partial\left(\rho c_{p} u_{y} T\right)}{\partial y}=\frac{\partial}{\partial x}\left(k \frac{\partial T}{\partial x}\right)+\frac{\partial}{\partial y}\left(k \frac{\partial T}{\partial y}\right)
$$

where $\rho, c_{p}, k$ are, respectively, the density, the specific heat capacity, and the thermal conductivity of the fluid. In Plug $1, \rho=\rho_{1}, c_{p}=c_{p 1}$, and $k=k_{1}$, while in Plug 2, $\rho=\rho_{2}, c_{p}=c_{p 2}$, and $k=k_{2}$.

Using the properties of Plug 1 as the characteristic values, Eq. (3) could be non-dimensionalized to

$$
\frac{\partial\left(\widehat{\rho c_{p}} \hat{T}\right)}{\partial \hat{t}}+\frac{\partial\left(\widehat{\rho c}_{p} \hat{u}_{x} \hat{T}\right)}{\partial \hat{x}}+\frac{\partial\left(\widehat{\rho c_{p}} \hat{u}_{y} \hat{T}\right)}{\partial \hat{y}}=\frac{1}{P e}\left[\frac{\partial}{\partial \hat{x}}\left(\hat{k} \frac{\partial \hat{T}}{\partial \hat{x}}\right)+\frac{\partial}{\partial \hat{y}}\left(\hat{k} \frac{\partial \hat{T}}{\partial \hat{y}}\right)\right]
$$

where $\hat{t} \equiv t / \tau, \tau \equiv h / V, \hat{T} \equiv\left(T-T_{0}\right) / T_{c}, \widehat{\rho c_{p}} \equiv \rho c_{p} /\left(\rho_{1} c_{p 1}\right), \hat{k} \equiv k / k_{1}$, $P e \equiv h V / \alpha_{1}, \quad \alpha_{1} \equiv k_{1} /\left(\rho_{1} c_{p 1}\right) . \quad \tau$ is the characteristic time, $\rho c_{p}$ is the volumetric heat capacity, and $P e$ is the Peclet number. With this normalization, the dimensionless time $\hat{t}$ and the dimensionless axial location of the plug train in the microchannel $\hat{X}$ satisfy,

$$
\hat{X}=\hat{t}
$$


i.e., at the instant $\hat{t}$, the plug train is at $\hat{X}=\hat{t}$ in the microchannel.

The ratio of the volumetric heat capacities between the two plugs is defined as

$$
\xi \equiv \frac{\rho_{2} c_{p 2}}{\rho_{1} c_{p 1}}
$$

while the ratio of the thermal conductivities is defined as

$$
\kappa \equiv k_{2} / k_{1}
$$

Two types of boundary conditions for heat transfer are considered, as shown in Figure 1: (1) asymmetric constant surface temperature boundary condition [abbreviated to TA, Figure 1(a)], and (2) asymmetric constant surface heat flux boundary condition [abbreviated to QA, Figure 1(b)]. For the TA boundary condition, the characteristic temperature $T_{c}$ is defined as

$$
T_{c}=T_{\mathrm{w}}-T_{0}
$$

Therefore, the corresponding boundary conditions at the channel walls in dimensionless forms are

$$
\begin{aligned}
& \frac{\partial \hat{T}}{\partial \hat{y}}=0 \text { at } \hat{y}=1 \\
& \hat{T}=1 \text { at } \hat{y}=-1
\end{aligned}
$$

For the QA boundary condition [Figure 1(b)], the characteristic temperature $T_{c}$ is defined as

$$
T_{c}=q^{\prime \prime}{ }_{\mathrm{w}} / k_{1}
$$

where $q^{\prime \prime}{ }_{\mathrm{w}}$ is the heat flux across the channel wall. The corresponding boundary conditions in dimensionless forms are

$$
\frac{\partial \hat{T}}{\partial \hat{y}}=0 \text { at } \hat{y}=1
$$




$$
\frac{\partial \hat{T}}{\partial \hat{y}}=1 \text { at } \hat{y}=-1
$$

In both the TA and QA boundary conditions, at the interfaces of the two plugs, the temperatures of the two adjacent plugs are identical, and the energy is conserved:

$$
\begin{aligned}
\left.k_{1} \frac{\partial \hat{T}}{\partial \hat{x}}\right|_{\hat{x}_{1}=1} & =\left.k_{2} \frac{\partial \hat{T}}{\partial \hat{x}}\right|_{\hat{x}_{2}=-1} \\
\left.\hat{T}\right|_{\hat{x}_{1}=1} & =\left.\hat{T}\right|_{\hat{x}_{2}=-1} \\
\left.k_{2} \frac{\partial \hat{T}}{\partial \hat{x}}\right|_{\hat{x}_{2}=1} & =\left.k_{1} \frac{\partial \hat{T}}{\partial \hat{x}}\right|_{\hat{x}_{1}=-1} \\
\left.\hat{T}\right|_{\hat{x}_{2}=1} & =\left.\hat{T}\right|_{\hat{x}_{1}=-1}
\end{aligned}
$$

The finite volume method (FVM) [37] was used to solve Eq. (4) for the unsteady heat transfer in plug trains. The simulation domain, i.e., two consecutive plugs, was divided into structured control volumes (CVs) with uniform size. A staggered grid is adopted to avoid the check-board distribution of the pressure field. The transient term was discretised using first order implicit scheme, the convection term using the power law scheme, while the diffusion term using the second order central difference scheme. In the iterative produce, the solution is considered converged if the maximum change of the dimensionless temperature for two successive iterations is less than a prescribed value $10^{-10}$,

$$
\left|\hat{T}^{n}-\hat{T}^{n+1}\right|<10^{-10}
$$

Grid independency study was performed by the repeating refinement of the grid and the time step until grid independent solutions were obtained, as 


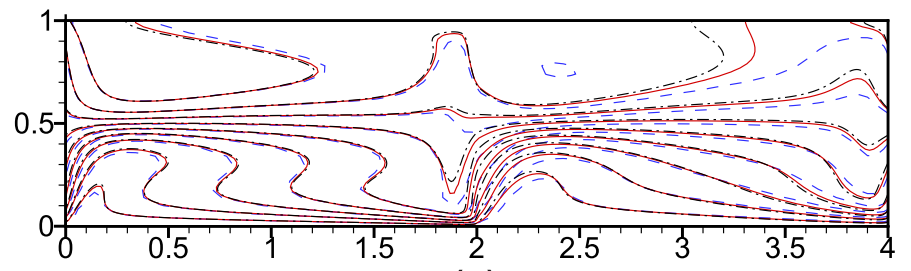

(a)

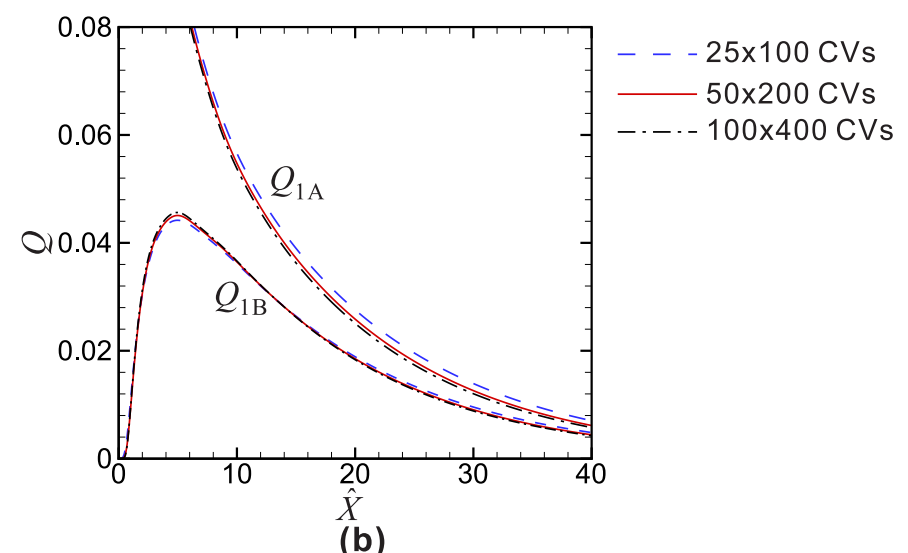

Figure 3: Grid independency study for heat transfer in plug train flow. Three computational grids are compared here, namely $25 \times 100,50 \times 200,100 \times 400 \mathrm{CVs}$, while the time steps $\Delta t$ are $4 \times 10^{-2}, 2 \times 10^{-2}, 1 \times 10^{-2}$, respectively. (a) Temperature contours at $\hat{X}=5$; (b) Heat rates $Q_{1 \mathrm{~A}}$ and $Q_{1 \mathrm{~B}}$ as described in Section 3.1.

shown in Figure 3. The comparison show that the grid with $50 \times 200 \mathrm{CVs}$ and the time step $\Delta t=2 \times 10^{-2}$ is grid independent for the simulation. The grid independency is also tested for different parameters, e.g., the conductivity ratio, the dimensionless plug length. Therefore, this grid density is sufficient for the problem of plug train heat transfer at different parameters. 


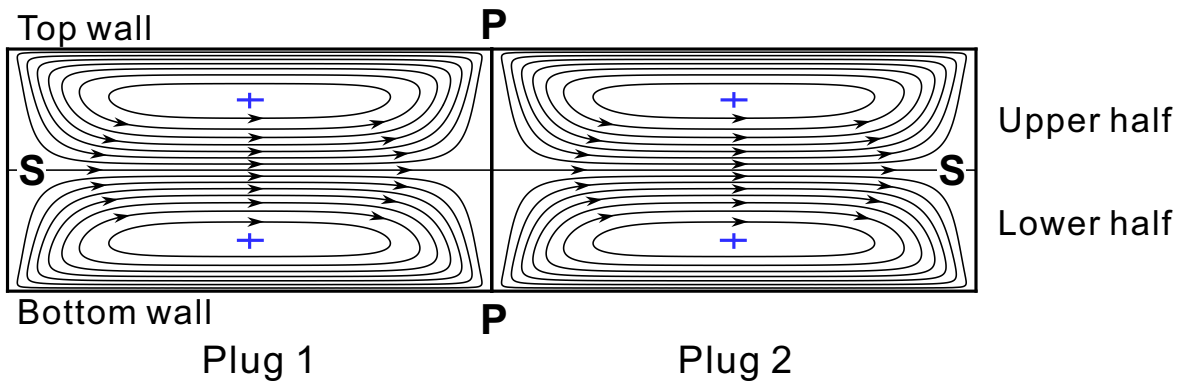

Figure 4: Flow pattern in a typical plug train. The dimensionless lengths are $\hat{L}_{1}=\hat{L}_{2}=2$, and the viscosity ratio of the two plugs is $\mu_{2} / \mu_{1}=1$. The mid-plane of the plug train is indicated by $\mathbf{S S}$, and the plug-plug interface is indicated by $\mathbf{P P}$.

\section{Results and discussion}

\subsection{Flow patterns in plug trains}

The flow pattern in a typical liquid plug is shown in Figure 4. Counterrotating vortices being symmetric about the mid-plane SS $(\hat{y}=0)$ are formed in each plug. Fluid in each half (the lower half and the upper half) of the plug are mixed, but the two halves remain unmixed and separated by the mid-plane SS. Heat transfer from the lower half to the upper half relies on thermal diffusion across the mid-plane. Therefore, the mid-plane SS serves as a barrier of heat transfer in liquid plugs. In addition, due to the presence of the interfaces between the two plugs (PP in Figure 4), heat transfer across the plug-plug interfaces also relies on thermal diffusion.

The schematic diagram of heat transfer in plug trains with asymmetric boundary conditions is illustrated in Figure 5. Thermal energy is absorbed by the liquid in the region near the bottom heated wall, advected by the recirculating flow within the lower half of the plug, then diffused across the mid-plane SS, and finally carried by the recirculating flow in the upper half 


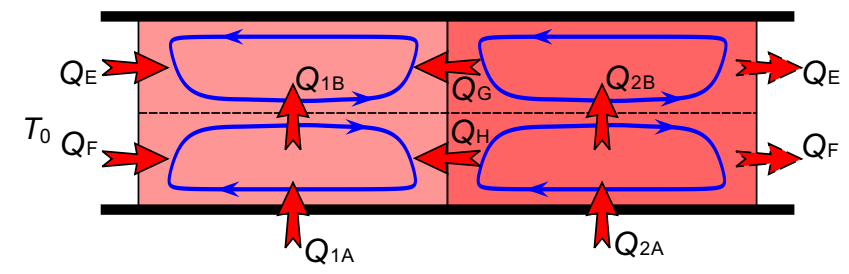

Figure 5: Schematic diagram of heat flow for asymmetric heat transfer in plug trains.

of the liquid plugs. At the same time, heat is diffused to the adjacent plugs at the plug-plug interfaces (PP).

To quantify the heat transfer at the bottom wall, across the plug-plug interface (PP), and across the mid-plane (SS), the following quantities are defined, as shown in Figure 5. $Q_{1 A}$ and $Q_{2 A}$ refer to the heat rates at the bottom wall over Plug 1 and Plug 2, respectively. $Q_{1 B}$ and $Q_{2 B}$ refer to the heat rates across the mid-plane (SS) in Plug 1 and Plug 2, respectively. $Q_{G}$ and $Q_{H}$ refer to the heat rates cross the plug-plug interface at the upper and lower halves, respectively. $Q_{E}$ and $Q_{F}$ refer to the heat rates across the rear interface of Plug 1 at upper and lower halves, respectively, and also periodically at the front interface of Plug 2. For simplicity, the heat rates are presented in dimensionless forms, i.e.,

$$
Q=-\hat{k} \frac{\partial \hat{T}}{\partial \hat{n}}
$$

where $\hat{n}$ is the unit directional vector normal to the plane as indicated by the arrows in Figure 5. 


\subsection{Heat transfer with asymmetric constant surface temperature (TA) bound-}

ary condition

\subsubsection{Process of asymmetric heat transfer}

The process of symmetric heat transfer in a single plug can be divided into three stages [10,11], (i) development of thermal boundary layer, (ii) advection of heated/fresh fluid in the plug, and (iii) thermally fully developed flow. For asymmetric heat transfer in plug trains, the process is different due to (i) the asymmetric boundary condition on the top/bottom walls and (ii) the coupling effect with adjacent plugs.

The heat transfer process with TA boundary condition in a typical plug train is shown in Figure 6. The bottom wall of the microchannel is maintained at a constant surface temperature $\left(\hat{T}_{\mathrm{w}}=1\right)$, while the top wall is adiabatic.

Initially, the plug train has a uniform low temperature $\left(\hat{T}_{0}=0\right)$ [Figure 6(a)]. When the plug train enters the channel, it begins to absorb heat from the bottom wall. Unlike the symmetric case [10], in the asymmetric boundary condition, a thermal boundary layer is formed only at the bottom wall [Figure 6(b)], and the thickness of the thermal boundary layer increases with time.

After the development of the thermal boundary layer, the recirculating flow in the lower half of the plug carries the heated fluid towards the central region of the lower half of the plug, while the upper half is still at the initial temperature [Figure 6(c)]. With the formation of temperature gradient across the mid-plane (SS), heat is diffused from the lower half of the plug to the upper half [Figure 6(d)]. Therefore, the process of heat transfer is strongly affected by thermal diffusion across the mid-plane (SS). After that, 
the temperature in the upper half of the plug gradually increases as heat is convected by the recirculating flow in the upper half [Figure 6(e)].

In this way, the convection in the lower half, the diffusion across the midplane $\mathbf{S S}$, and the convection in the upper half gradually homogenise the fluid temperature in the plug. The temperature difference between the fluid and the bottom wall gradually decreases.

As the heat transfer process goes on, the pattern of the temperature contour does not change with time, and the heat transfer process reaches a fully developed stage [Figure 6(f)]. In this stage, the pattern of the temperature contours is not symmetric about the mid-plane (SS), and temperature difference exists between the lower half and the upper half, as shown in Figure 6(f).

\subsubsection{Heat transfer across the bottom wall and across the mid-plane $\boldsymbol{S} \boldsymbol{S}$}

To quantitatively investigate the mechanisms of asymmetric heat transfer in plug trains, the heat rates at the bottom wall and at the mid-plane SS are plotted in Figure 7. For Plug 1, there is a large temperature difference between the wall and the liquid initially. Therefore, a large value of $Q_{1 A}$ is formed by thermal conduction over the wall. At the same time, $Q_{1 B}$ is zero because the fluid in the middle region still remains at the initial temperature. As time passes, $Q_{1 A}$ decreases dramatically because of the development of the thermal boundary layer and the increase of the fluid temperature in the lower half of the plug. The increase of the fluid temperature in the lower half also results in the increase of $Q_{1 B}$, as shown in Figure 7. Therefore, the fluid temperature in the upper half gradually increases, which resists the increase of $Q_{1 B}$. Consequently, $Q_{1 B}$ decreases gradually after reaching a maximum. 
As heat transfer progresses, the fluid temperature gradually approaches the wall temperature, and the heat rates, $Q_{1 A}$ and $Q_{1 B}$, gradually approach 0 .

The heat rates in Plug 2, $Q_{2 A}$ and $Q_{2 B}$, have the similar trends to those in Plug 1 but have relatively larger magnitudes. This is because Plug 2 has a larger thermal conductivity than Plug 1 with a ratio of $\kappa=2$.

\subsubsection{Heat transfer across plug-plug interface $\boldsymbol{P P}$}

The coupling effect between plugs results in heat transfer between adjacent plugs, affecting the overall process of asymmetric heat transfer in plug trains. The heat rates at plug-plug interfaces are plotted in Figure 7(b). Taking Plug 1 as an example, during the formation of the thermal boundary layer, $Q_{F}<0$ indicates that heat transfer is from Plug 1 to the adjacent plug at the rear interface of Plug 1, while $Q_{H}>0$ indicates that heat transfer is from Plug 2 to Plug 1 at the front interface of Plug 1. This is because the recirculating flow in the lower half of the plug is in the clockwise direction. The thicker thermal boundary layer at the rear of the plug results in a higher fluid temperature than that at the front. The magnitudes of $Q_{F}$ and $Q_{H}$ increase dramatically with the development of the thermal boundary layer. This is because the growth of the thermal boundary layer results in a larger temperature difference between the front and the rear of the plug.

The advection of the heated fluid in the lower half of Plug 1 homogenize the fluid temperature, which reduces the heat transfer at plug-plug interfaces $Q_{F}$ and $Q_{H}$. As time passes, the fluid temperature gradually approaches the wall temperature. Consequently, the heat transfer rates at the plug interfaces, $Q_{F}$ and $Q_{H}$, gradually approach 0 .

The other two paths of heat flow between plugs is at the upper part of the 


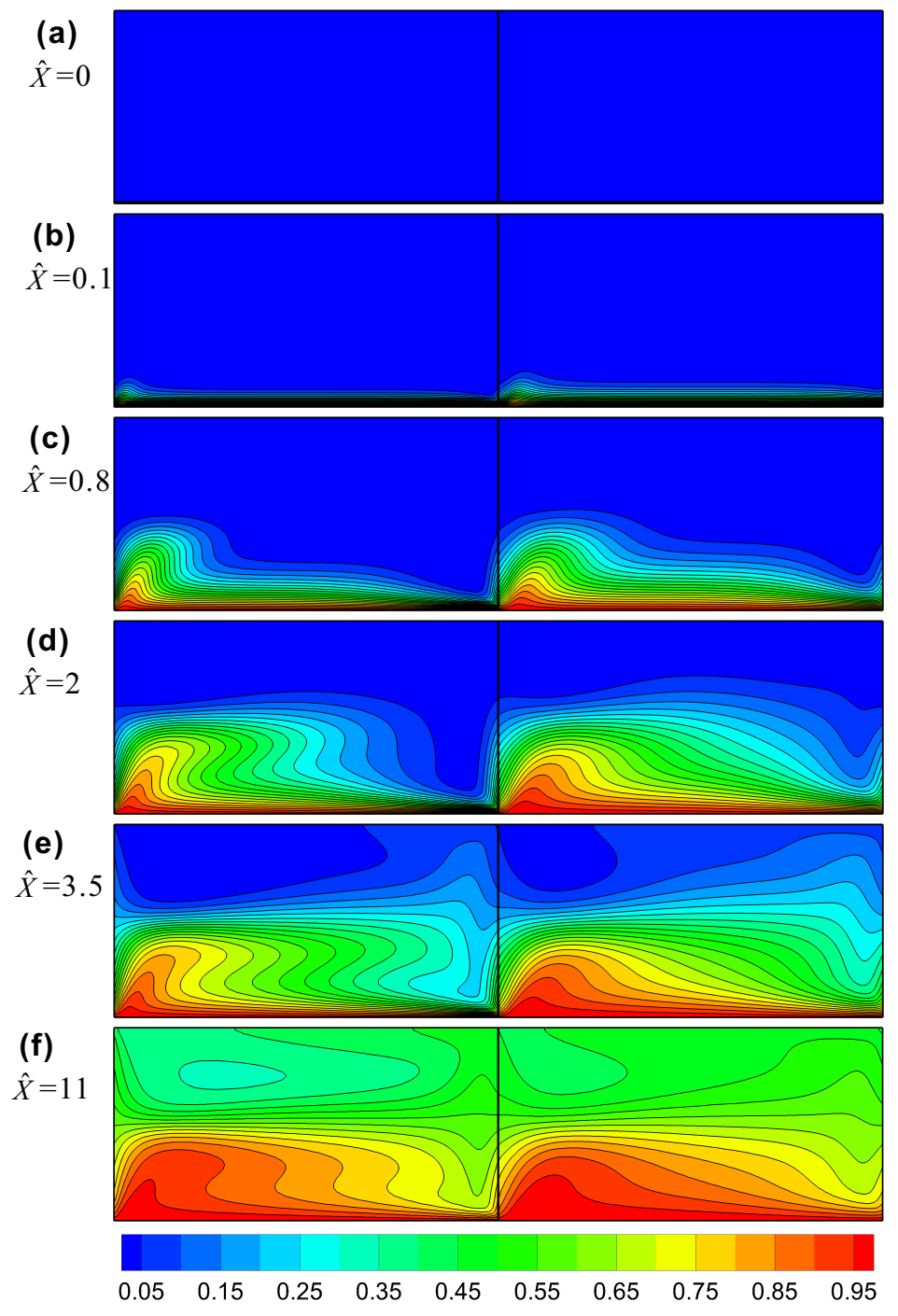

Figure 6: Evolution of temperature contours in plug trains with TA boundary condition. The locations of the plug trains $\hat{X}$ are, respectively, (a) 0, (b) 0.1, (c) 0.8, (d) 2, (e) 3.5, and (f) 11. The dimensionless lengths of the plugs are $\hat{L}_{1}=\hat{L}_{2}=2$. The ratio of the volumetric heat capacities between the two plugs is $\xi=1$, while the ratio of the thermal conductivities is $\kappa=2$. The Peclet number is $P e=100$. 


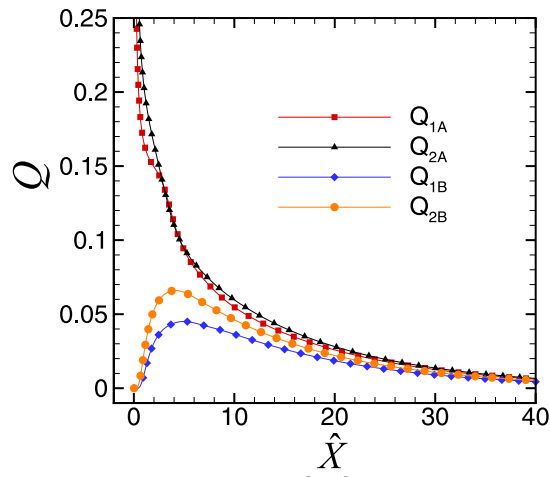

(a)

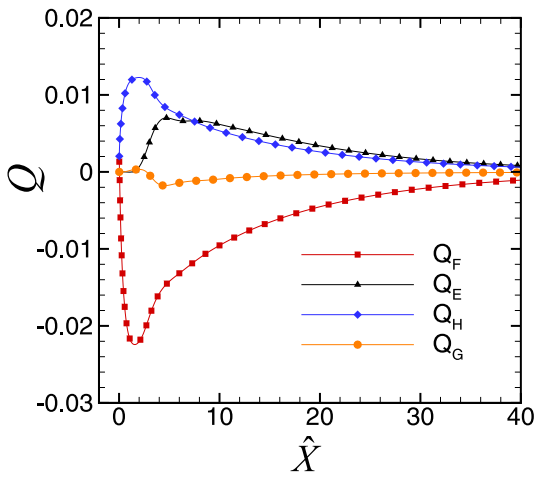

(b)

Figure 7: Variation of the heat rates at different planes of the plug train against the axial location of the channel. (a) Heat rates across the bottom wall and the mid-plane $Q_{1 \mathrm{~A}}$, $Q_{2 \mathrm{~A}}, Q_{1 \mathrm{~B}}$, and $Q_{2 \mathrm{~B}}$. (b) Heat rates across plug-plug interfaces $Q_{\mathrm{E}}, Q_{\mathrm{F}}, Q_{\mathrm{G}}$, and $Q_{\mathrm{H}}$. The parameters of flow and heat transfer are identical to those in Figure 6.

plugs, $Q_{E}$ and $Q_{G}$. Initially, $Q_{E}$ and $Q_{G}$ are 0, as shown in Figure 7(b). This is because the fluid in the upper region is still at the initial temperature. As the heat transfer progress, the fluid temperature in the upper half of the plug increases. Due to the counter-clockwise recirculating flow in the upper half of the plug, the fluid near the front has a higher temperature than that at the rear of the plug. Therefore, at the front of Plug 1, heat is transferring from Plug 1 to Plug $2\left(Q_{G}<0\right)$, while at the rear of Plug 1, heat is transferring from the adjacent plug to Plug $1\left(Q_{E}>0\right)$. As the temperature difference between the front region and the rear region builds up, the magnitudes of $Q_{E}$ and $Q_{G}$ increase, which indicate the increase of heat rate at the interfaces. As the fluid temperature gradually approaches the wall temperature, the magnitudes of $Q_{E}$ and $Q_{G}$ gradually decrease and approach 0 . 


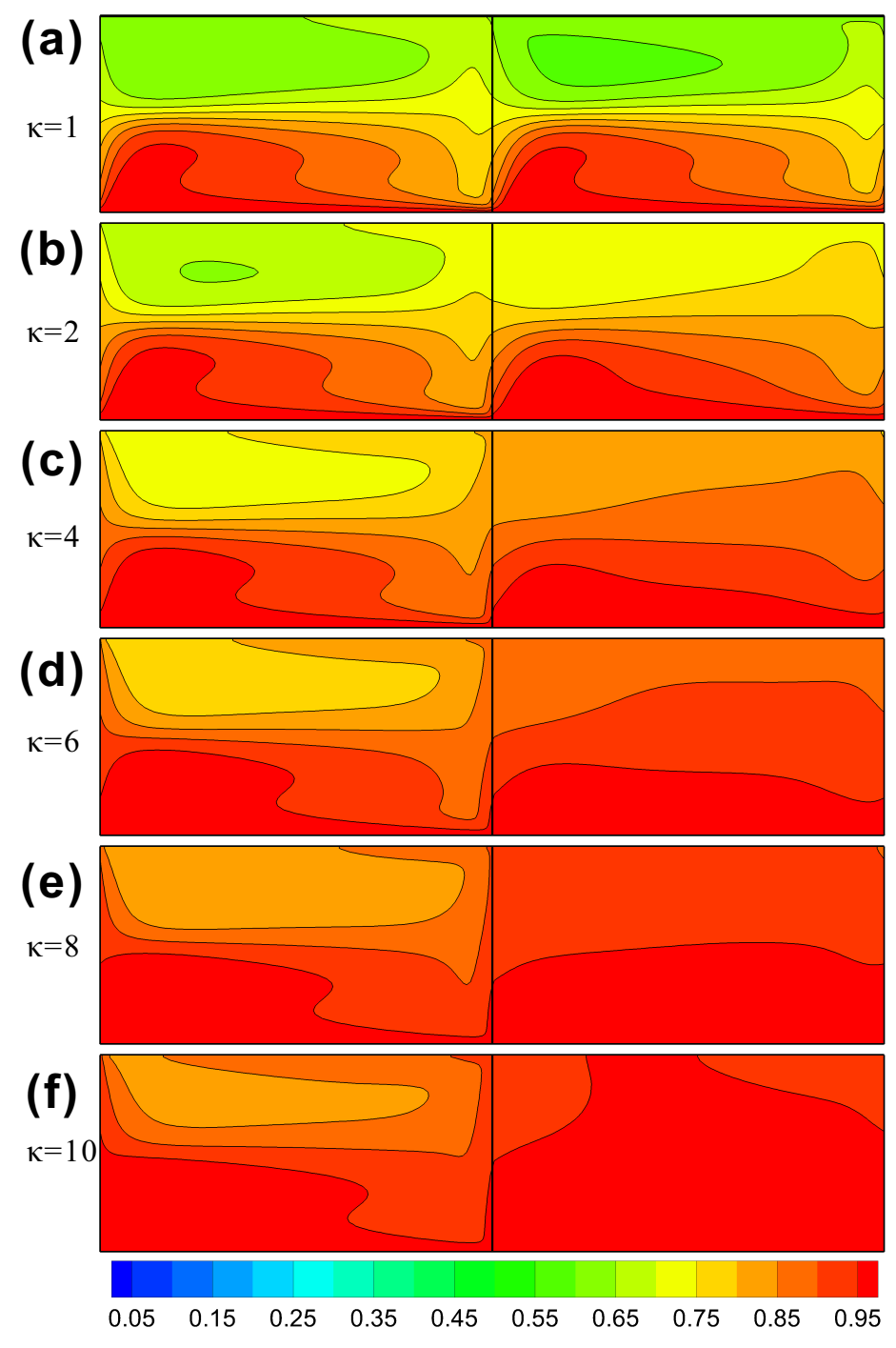

Figure 8: Temperature contours for plug trains at different ratios of thermal conductivity ((a) $\kappa=1$, (b)2, (c) 4, (d) 6 , (e) 8, (f) 10) at the axial location of $\hat{X}=20$ with TA boundary condition. The dimensionless lengths of the plugs are $\hat{L}_{1}=\hat{L}_{2}=2$. The ratio of the volumetric heat capacities is $\xi=1$. The Peclet number is $P e=100$. 

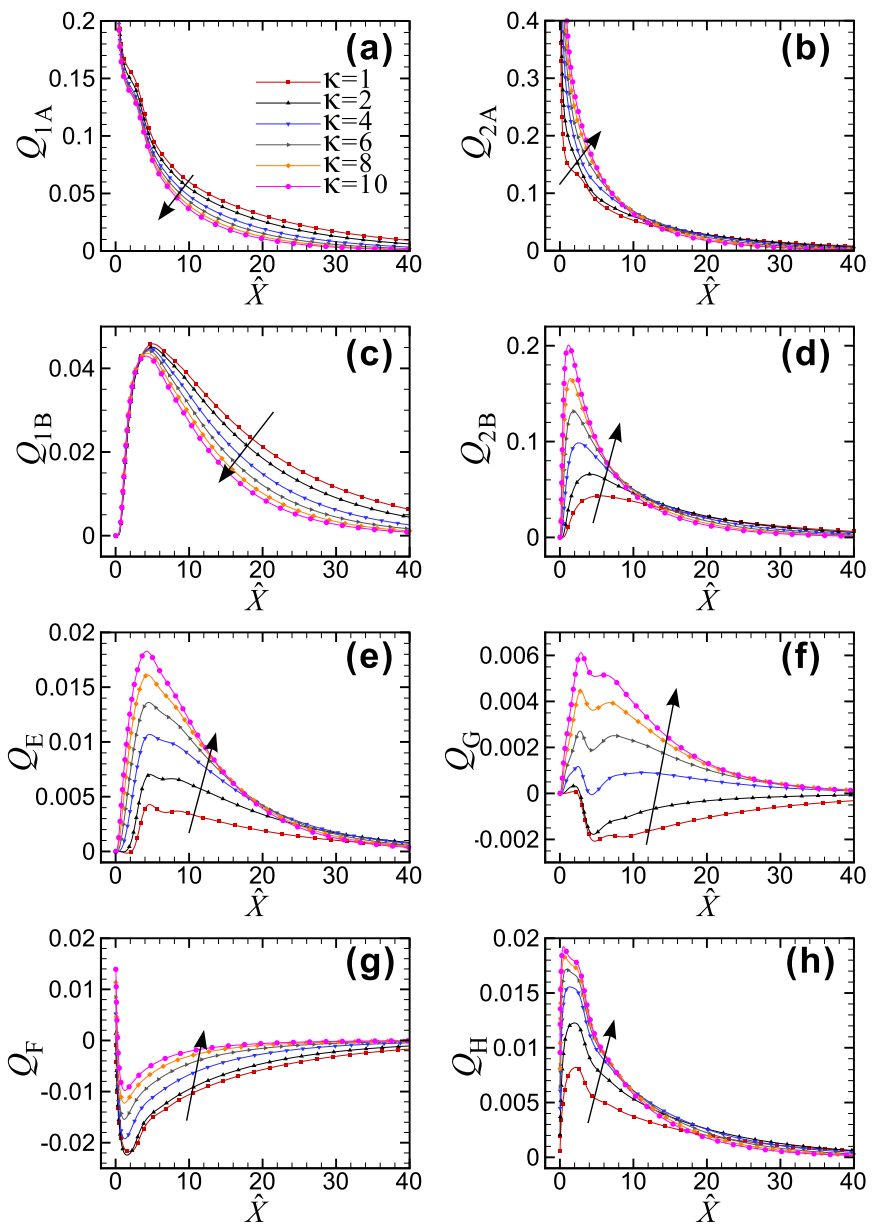

Figure 9: Heat rates for plug trains of different ratios of thermal conductivity (a) $Q_{1 \mathrm{~A}}$, (b) $Q_{2 \mathrm{~A}}$, (c) $Q_{1 \mathrm{~B}}$, (d) $Q_{2 \mathrm{~B}}$, (e) $Q_{\mathrm{E}}$, (f) $Q_{\mathrm{G}}$, (g) $Q_{\mathrm{F}}$, and (h) $Q_{\mathrm{H}}$. The arrows in the figures are the direction of increasing $\kappa$. The parameters of flow and heat transfer are the same as those in Figure 8. 


\subsubsection{Effect of conductivity ratio $(\kappa)$}

To study the effect of conductivity ratio $(\kappa)$ on the process of asymmetric heat transfer, the thermal conductivity of Plug $1\left(k_{1}\right)$ was fixed, while the thermal conductivity of Plug $2\left(k_{2}\right)$ was varied to achieve a conductivity ratio $(\kappa)$ ranging from 1 to 10 . The temperature contours at the axial location $\hat{X}=20$ are plotted in Figure 8. With increasing $\kappa$ (i.e., increasing $k_{2}$ ), more heat is transferred into Plug 2. Therefore, Plug 2 reaches a higher and more uniform temperature than Plug 1. In contrast, the temperature distribution in Plug 1 is less uniformed and dominated by advection.

The heat rates across different planes are plotted against the axial location of the microchannel in Figure 9. With increasing $\kappa$, heat transfer over the wall of Plug $2\left(Q_{2 A}\right)$ increases because a higher conductivity in Plug 2 conducts more heat from the wall [Figure 9(b)]. Consequently, the heat transfer process at the mid-plane SS over Plug $2\left(Q_{2 B}\right)$ also increases [Figure 9(d)].

Since the heat transfer process to Plug 2 is enhanced by increasing $\kappa$, more heat can flow from Plug 2 to Plug 1 through the plug-plug interface PP. Consequently, $Q_{1 A}$ reduces as $\kappa$ increases [Figure 9(a)]. Similarly, with increasing heat transfer at the interface, the heat rate through the mid-plane SS in Plug 1 reduces [Figure 9(c)].

With increasing $\kappa$, Plug 2 reaches a higher temperature than Plug 1 due to the larger $k_{2}$. Therefore, more heat is conducted through the plugplug interfaces [Figures 9(h) and (f)]. Note that increasing $\kappa$ can change the direction of the heat transfer at the upper part of the interface (from $Q_{G}<0$ to $Q_{G}>0$ ). In addition, at the rear interface of Plug 1, the 
increased temperature of the adjacent plug causes $Q_{F}$ to reduce in magnitude [Figure $9(\mathrm{~g})]$. The heat transfer rate at the upper part of the rear interface of Plug 1 also increases [Figure 9(e)].

\subsubsection{Effect of plug length}

The effect of plug length on the heat transfer in plug trains is studied by varying the dimensionless length of Plug $2, \hat{L}_{2}$, from 1 to 4 while maintaining the length of Plug 1 at $\hat{L}_{1}=2$. The temperature contours at the axial location $\hat{X}=20$ are plotted in Figure 10 . Due to the presence of the interface, the central region of plugs is dominated by the parallel flow, while the front/rear regions are dominated by the transverse flow, as shown in Figure 4. Therefore, for long plugs, the parallel flow in the central region of Plug 2 produces parallel temperature contours. In contrast, for short plugs, the transverse flow in the front/rear can effectively mix the heated and fresh fluid in the plug and distort the temperature contours. Therefore, when $\hat{L}_{2}=1$, the heat transfer in Plug 2 is faster and the temperature distribution is more uniform than that at $\hat{L}_{2}=4$, as shown in Figure 10a.

In addition, due to the heat transfer across plug-plug interface, the higher temperature in the upper half of Plug 2 at $\hat{L}_{2}=1$ can provide more heat to Plug 1 than that at $\hat{L}_{2}=4$. Then, the temperature in the upper half of Plug 1 is higher than that at $\hat{L}_{2}=4$. Therefore, a short length of Plug 2 improves not only the heat transfer in Plug 2 but also the heat transfer in Plug 1, as shown in Figure 10a. 
(a)

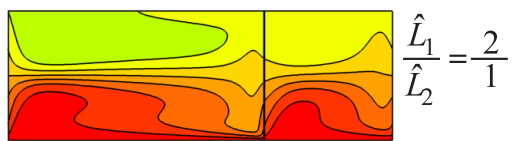

(b)

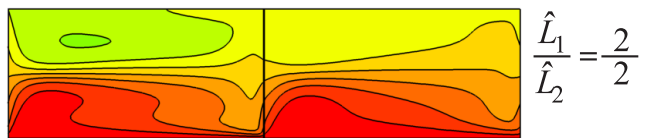

(c)

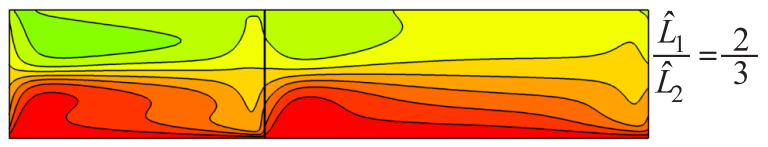

(d)

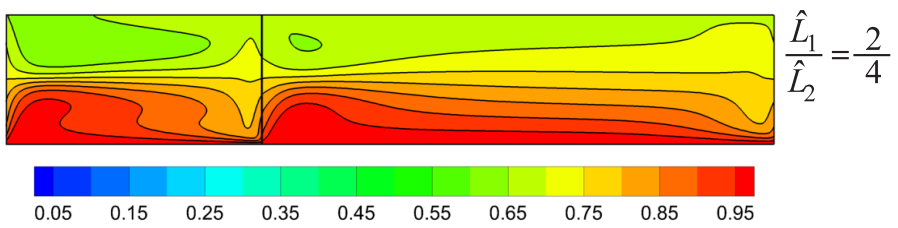

Figure 10: Temperature contours for plug trains of different lengths at the axial location of $\hat{X}=20$ with TA boundary condition. The dimensionless length of Plug 1 is fixed at $\hat{L}_{1}=2$, while the dimensionless length of Plug 2 are respectively (a) $\hat{L}_{2}=1$, (b) $\hat{L}_{2}=2$, (c) $\hat{L}_{3}=3$, and (d) $\hat{L}_{2}=4$. The ratio of the volumetric heat capacities is $\xi=1$, the ratio of thermal conductivity is $\kappa=2$, and the Peclet number is $P e=100$. 


\subsubsection{Effect of Peclet number}

To study the effect of Peclet number on heat transfer in plug trains, the Peclet number is increased from 50 to 6400, while other parameters are fixed,

i.e., the dimensionless plug length $\hat{L}_{1}=\hat{L}_{2}=2$, the ratio of the volumetric heat capacities $\xi=1$, and the ratio of thermal conductivity $\kappa=2$. The temperature contours at the axial location $\hat{X}=20$ are shown in Figure 11. At a low Peclet number (e.g., $P e=50$ in Figure 11a), the diffusion is strong, and heat is diffused rapidly from the bottom wall to the entire region of the plugs. As the Peclet number increases (e.g., $P e=6400$ in Figure 11e), the diffusion effect reduces, and the advection effect becomes prominent, which advects the heated fluid along the streamlines. In addition, due to the low diffusion effect at high Peclet numbers, large temperature gradient is formed near the bottom wall and near the mid-plane SS, which indicates the inefficient heat transfer from the bottom wall to the lower half of the plug and from the lower half to the upper half of the plug.

\subsection{Heat transfer with $Q A$ boundary condition}

\subsubsection{Process of asymmetric heat transfer}

The heat transfer at QA boundary condition shares similar features with that at TA boundary condition, as shown in Figure 12. The bottom wall is maintained at a constant surface heat flux, the top wall is adiabatic, and the plug initially has a uniform temperature [Figure 12(a)]. A thermal boundary layer is formed immediately at the bottom wall of the microchannel [Figure 12(b)], and its thickness increases with the development of the thermal boundary layer. The recirculating flow advects the heated fluid in the lower half of the plug, and the thermal conduction diffuses heat from 


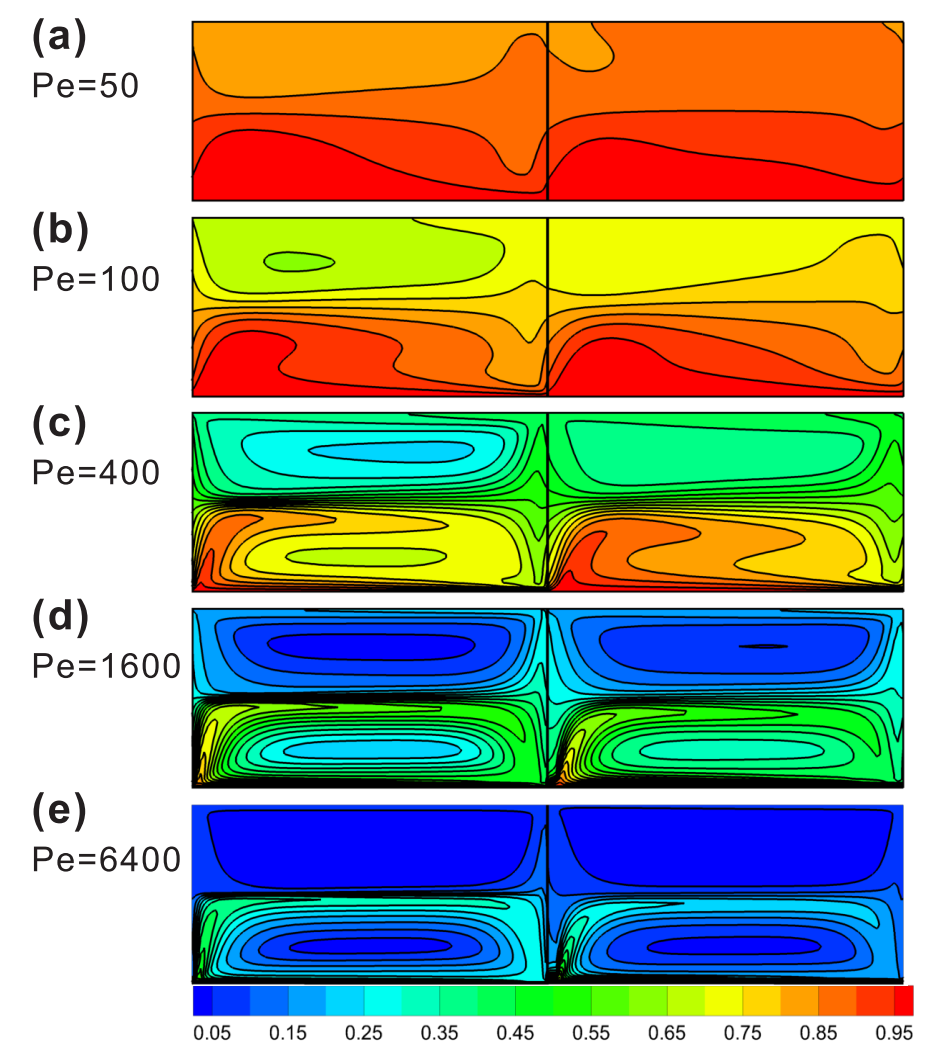

Figure 11: Temperature contours for plug trains at different Peclet numbers at the axial location of $\hat{X}=20$ with TA boundary condition. The Peclet numbers are respectively (a) $P e=50$, (b) $P e=100$, (c) $P e=400$, (d) $P e=1600$, and (e) $P e=6400$. The dimensionless plug lengths are at $\hat{L}_{1}=\hat{L}_{2}=2$. The ratio of the volumetric heat capacities is $\xi=1$, the ratio of thermal conductivity is $\kappa=2$. 


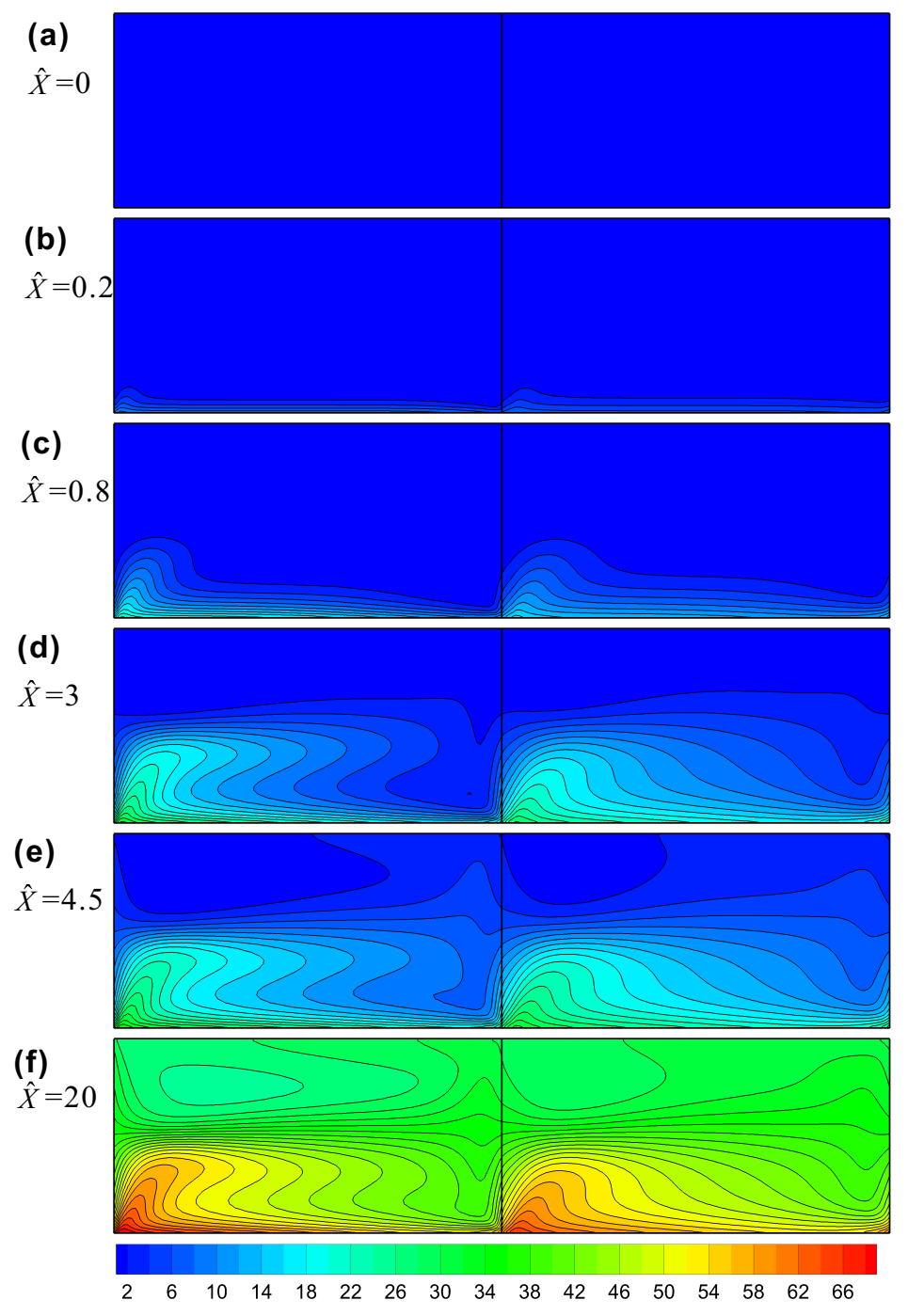

Figure 12: Evolution of temperature contours of heat transfer in plug trains at QA boundary condition. The locations of the plug trains $\hat{X}$ are, respectively, (a) 0 , (b) 0.2 , (c) 0.8 , (d) 3, (e) 4.5, and (f) 20. The dimensionless lengths of the plugs are $\hat{L}_{1}=\hat{L}_{2}=2$. The ratio of the volumetric heat capacity is $\xi=1$ and the ratio of thermal conductivity is $\kappa=2$. The Peclet number is $P e=100$. 
the lower half of the plug to the upper half. The recirculating flow also advects the heated fluid in the upper half of the plug. Figure 12(f) shows the temperature contours in the thermally fully developed stage. The fluid temperature does not approach any uniform temperature but keeps rising as heat is continuously absorbed from the bottom wall.

The asymmetric boundary condition results in heat transfer across the mid-plane, which is quantified by $Q_{1 B}$ and $Q_{2 B}$. Meanwhile, the coupling effect between adjacent plugs results in heat transfer across plug-plug interfaces, is quantified by $Q_{E}, Q_{F}, Q_{G}$, and $Q_{H}$, as illustrated in Figure 5.

\subsubsection{Heat transfer across the mid-planes}

With a constant surface heat flux maintained at the bottom walls, the heat rate at the bottom wall is uniform as shown in Figure 13(a). The heat rate across the mid-plane of Plug $1 Q_{1 B}$ is initially 0 due to the uniform temperature distribution. With the progress of heat transfer, the temperature in the lower half of the plug increases, and results in a temperature difference between the lower and upper half of Plug 1 . Therefore, a heat rate $Q_{1 B}$ is formed, which increases when the temperature difference gradually builds up. As the heat transfer in the plug train becomes fully developed, the heat rate $Q_{1 B}$ becomes constant.

The heat rates in Plug 2 have the same trend but of a larger magnitude. This is because Plug 2 has a larger thermal conductivity $(\kappa=2)$.

\subsubsection{Heat transfer across plug-plug interfaces}

The heat transfer across the plug-plug interfaces is quantified by $Q_{E}, Q_{F}$, $Q_{G}$, and $Q_{H}$, and plotted in Figure 13(b). Initially, $Q_{E}, Q_{F}, Q_{G}$, and $Q_{H}$ 


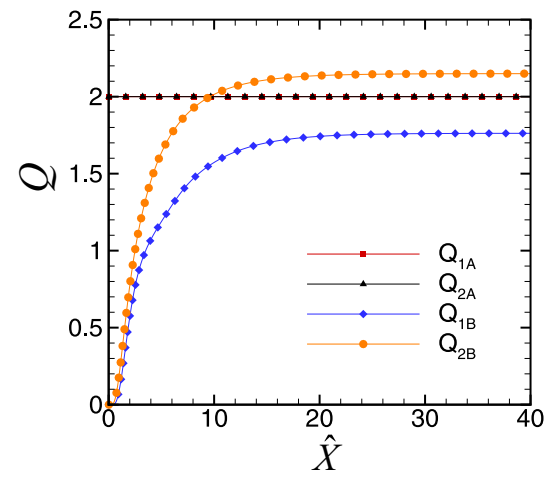

(a)

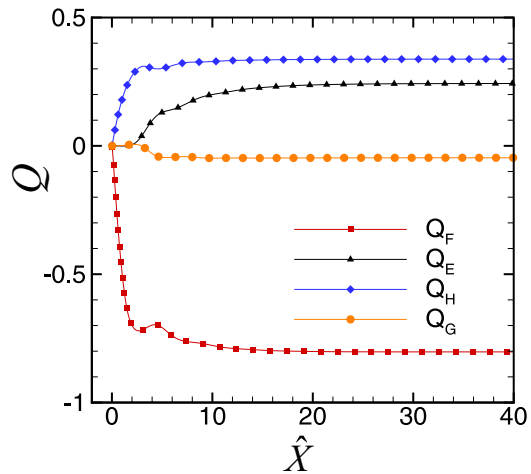

(b)

Figure 13: (a) Heat rates across the bottom wall and the mid-plane. (b) Heat flux across plug-plug interfaces. The parameters of flow and heat transfer are the same as those in Figure 12 .

are all zero because the temperature in the plug is uniform. The signs of these values are the same as those at TA boundary condition because the same recirculating direction results in the same direction of heat transfer. The magnitudes of $Q_{F}$ and $Q_{H}$ increase due to the built up of temperature difference in the plugs. The variation of $Q_{E}$ and $Q_{G}$ is delayed because it needs a certain period for heat transfer to the upper half of the plugs. A significant difference with TA boundary condition is that the heat rates at the interfaces do not approach 0 but constant values. This is because heat is continuously provided from the bottom wall, the fluid temperature in the plug keeps increasing, and the temperature gradient in the plug is maintained.

\subsubsection{Effect of conductivity ratio $(\kappa)$}

To study the effect of the ratio of thermal conductivities, the thermal conductivity of Plug 1 was fixed while the thermal conductivity of Plug 2 


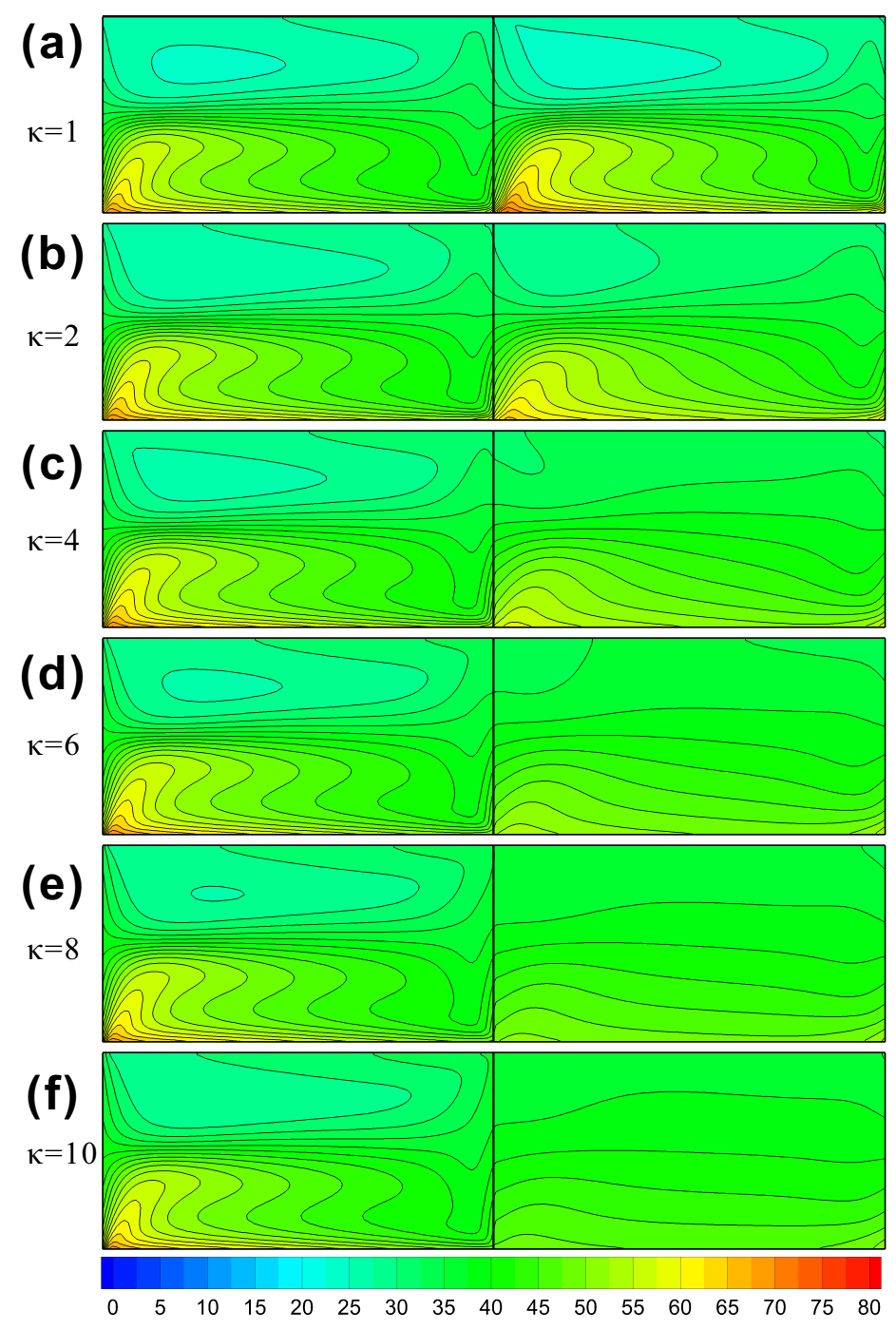

Figure 14: Temperature contours for plug trains with different ratios of thermal conductivities ((a) $\kappa=1$, (b) 2, (c) 4, (d) 6, (e) 8, (f) 10) at the axial location of $\hat{X}=20$ with QA boundary condition. The dimensionless lengths of the plugs are $\hat{L}_{1}=\hat{L}_{2}=2$. The ratio of the volumetric heat capacities is $\xi=1$. The Peclet number is $P e=100$. 

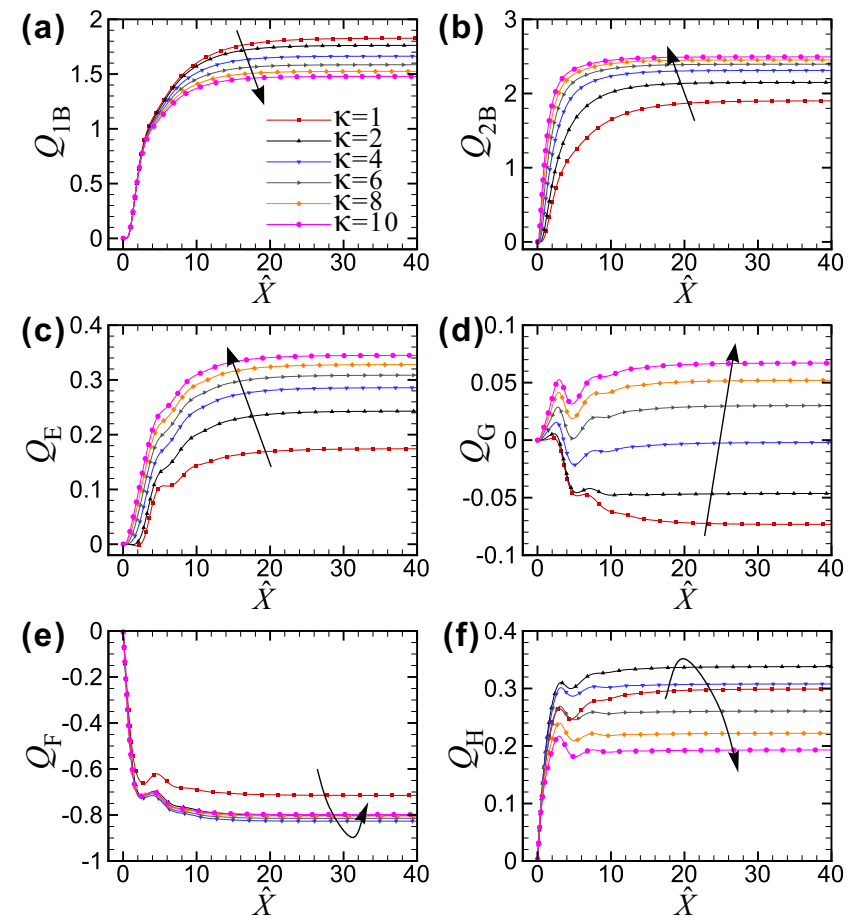

Figure 15: Heat rates at different planes for plug trains of different ratios of thermal conductivity $(\kappa=1,2,4,6,8,10)$. (a) $Q_{1 \mathrm{~B}}$, (b) $Q_{2 \mathrm{~B}}$, (c) $Q_{\mathrm{E}}$, (d) $Q_{\mathrm{G}}$, (e) $Q_{\mathrm{F}}$, and (f) $Q_{\mathrm{H}}$. The arrows in the figures are the direction of increasing $\kappa$. The parameters of flow and heat transfer are the same as those in Figure 14. 
was varied. The temperature contours at the axial location of $\hat{X}=20$ is shown in Figure 14, for the ratio of thermal conductivities ranging from 1 to 10. At a higher value of $\kappa$, the temperature in Plug 2 is more uniform, and is less distorted by the recirculating vortices. This is because the higher conductivity in Plug 2 can diffuse heat more effectively.

The variation of the heat transfer rates at different planes against the axial location of the channel are plotted in Figure 15, for plug trains with different ratios of thermal conductivities. With increasing $\kappa$, the thermal conductivity of Plug 2 increases, which more effectively transfer heat from the wall to the liquid in Plug 2. Being similar to that of TA boundary condition, $Q_{2 B}$ increases with increasing the ratio of thermal conductivity [Figure 15]. This is because heat can be effectively transferred to the mid-plane SS at large thermal conductivities. At the same time, the heat rate across the mid-plane in Plug 1 is reduced because more heat can be transferred at the plug-plug interfaces [Figure 15(a)]

With increasing $\kappa$, higher conductivities in Plug 2 result in a more uniform temperature in Plug 2. Therefore, the fluid temperature in the upper half of Plug 2 is higher than that of Plug 1, which results in the increases of $Q_{G}$, i.e., the heat rate at the upper half of the interface. Like that in TA boundary condition, $Q_{G}$ in QA boundary condition can become positive for large ratios of thermal conductivities. In addition, heat transfer at the rear interface of Plug 1 also increases under the effect of the adjacent plug.

The effect of $\kappa$ on heat transfer rates $Q_{F}$ and $Q_{H}$ is complex because the heat flux at the bottom wall is fixed at a constant value. In contrast to TA boundary condition, higher conductivities do not result in a higher 
temperature, but result in more uniform temperature distribution, as shown in Figure 14. This is because the heat flux at the wall is fixed to a constant value.

With increasing $\kappa$, the heat rate at the lower part of the interface between the two plugs increases, which is the same as that in TA boundary condition. However, since the heat flux at the bottom wall is fixed at a constant value, the heat provided by the bottom wall is immediately conducted away to the upper part of Plug 2, and the temperature gradient in the axial direction is greatly reduced. Therefore, the increasing of $\kappa$ cannot provide more heat for the heat transfer to Plug 1. Consequently, the heat transfer rate across the lower half of the interface $Q_{H}$ reduces with increasing $\kappa$. In addition, with increasing $\kappa$, the temperature distribution in the left adjacent plug is also very uniform like Plug 2. Therefore, the heat that is transferred to the left

adjacent plug could be effectively diffused away, which causes the increase of the magnitude of $Q_{F}$ with increasing $\kappa$. Also because a constant heat flux is maintained on the wall, the magnitude of $Q_{F}$ decrease slightly when $\kappa$ is increased above a certain limit.

\section{Conclusions}

The heat transfer process in plug trains subjecting to asymmetric boundary condition is investigated. Two types of boundary conditions are considered: asymmetric constant surface temperature, and asymmetric constant surface heat flux. The process of heat transfer in plug trains is analyzed and the paths of heat flow are identified. The effects of the thermal conductivity, the plug length, and the Peclet number on the heat 
transfer process and on the path of heat flow are studied. The results show that the heat transfer process with asymmetric boundary conditions is significantly different from that with symmetric boundary conditions. After the development of the thermal boundary layer, the recirculating flow in the lower half of the plug carries the heated fluid towards the central region of the lower half of the plug. With the formation of temperature gradient across the mid-plane, heat is diffused from the lower half of the plug to the upper half. The temperature in the upper half of the plug gradually increases as heat is convected by the recirculating flow in the upper half. In this way, the convection in the lower half, the diffusion across the mid-plane, and the convection in the upper half gradually transfer heat from the wall to the fluid in the plugs. Meanwhile, the process of heat transfer in the two adjacent plugs is coupled by heat transfer across plug-plug interfaces.

The results presented in this work can deepen our understanding of heat transfer in multiphase microfluidics, and also guide the design of microchannel heat sinks. The optimization of working conditions and selection of working fluids deserves more study by scientists and engineers in the future.

\section{Acknowledgements}

The authors gratefully acknowledge research support from the Singapore Ministry of Education Academic Research Fund Tier 2 Research Grant MOE2011-T2-1-036. 


\section{References}

[1] J. Kim, Spray cooling heat transfer: The state of the art, International Journal of Heat and Fluid Flow 28 (2007) 753-767.

[2] L. Lin, R. Ponnappan, Heat transfer characteristics of spray cooling in a closed loop, International Journal of Heat and Mass Transfer 46 (2003) $3737-3746$.

[3] Z. B. Yan, K. C. Toh, F. Duan, T. N. Wong, K. F. Choo, P. K. Chan, Y. S. Chua, Experimental study of impingement spray cooling for high power devices, Applied Thermal Engineering 30 (2010) 1225-1230.

[4] K. Jambunathan, E. Lai, M. A. Moss, B. L. Button, A review of heat transfer data for single circular jet impingement, International Journal of Heat and Fluid Flow 13 (1992) 106-115.

[5] S. Launay, V. Sartre, J. Bonjour, Parametric analysis of loop heat pipe operation: a literature review, International Journal of Thermal Sciences 46 (2007) 621-636.

[6] L. L. Vasiliev, Heat pipes in modern heat exchangers, Applied Thermal Engineering 25 (2005) 1-19.

[7] G. L. Morini, Single-phase convective heat transfer in microchannels: A review of experimental results, International Journal of Thermal Sciences 43 (2004) 631-651.

[8] N. T. Nguyen, D. Bochnia, R. Kiehnscherf, W. Dötzel, Investigation 
of forced convection in microfluid systems, Sensors and Actuators APhysical 55 (1996) 49-55.

[9] B. Palm, Heat transfer in microchannels, Microscale Thermophysical Engineering 5 (2001) 155-175.

[10] Z. Che, T. N. Wong, N.-T. Nguyen, Heat transfer enhancement by recirculating flow within liquid plugs in microchannels, International Journal of Heat and Mass Transfer 55 (2012) 1947-1956.

[11] Z. Che, T. N. Wong, N.-T. Nguyen, Heat transfer in plug flow in cylindrical microcapillaries with constant surface heat flux, International Journal of Thermal Sciences 64 (2013) 204-212.

[12] N. Kim, M. C. Murphy, S. A. Soper, D. E. Nikitopoulos, Liquid-liquid segmented flows in polycarbonate microchannels with cross-sectional expansions, International Journal of Multiphase Flow 58 (2014) 83 96.

[13] D. A. Hoang, V. van Steijn, L. M. Portela, M. T. Kreutzer, C. R. Kleijn, Benchmark numerical simulations of segmented two-phase flows in microchannels using the volume of fluid method, Computers \& Fluids 86 (2013) $28-36$.

[14] D. M. Fries, F. Trachsel, P. R. von Rohr, Segmented gas-liquid flow characterization in rectangular microchannels, International Journal of Multiphase Flow 34 (2008) 1108 - 1118.

[15] V. van Steijn, M. Kreutzer, C. Kleijn, Velocity fluctuations of segmented 
flow in microchannels, Chemical Engineering Journal 135, Supplement 1 (2008) S159 - S165.

[16] A. Asthana, I. Zinovik, C. Weinmueller, D. Poulikakos, Significant nusselt number increase in microchannels with a segmented flow of two immiscible liquids: An experimental study, International Journal of Heat and Mass Transfer 54 (2011) 1456 - 1464.

[17] V. Dore, D. Tsaoulidis, P. Angeli, Mixing patterns in water plugs during water/ionic liquid segmented flow in microchannels, Chemical Engineering Science 80 (2012) 334 - 341.

[18] J. Tan, Y. Lu, J. Xu, G. Luo, Mass transfer performance of gas-liquid segmented flow in microchannels, Chemical Engineering Journal 181-182 (2012) $229-235$.

[19] A. R. Betz, D. Attinger, Can segmented flow enhance heat transfer in microchannel heat sinks?, International Journal of Heat and Mass Transfer 53 (2010) 3683-3691.

[20] S. S. Y. Leung, Y. Liu, D. F. Fletcher, B. S. Haynes, Heat transfer in well-characterised taylor flow, Chemical Engineering Science 65 (2010) 6379-6388.

[21] Y. S. Lim, S. C. M. Yu, N. T. Nguyen, Flow visualization and heat transfer characteristics of gascliquid two-phase flow in microtube under constant heat flux at wall, International Journal of Heat and Mass Transfer 56 (2013) 350-359. 
[22] Y. S. Muzychka, E. Walsh, P. Walsh, Simple models for laminar thermally developing slug flow in noncircular ducts and channels, Journal of Heat Transfer 132 (2010) 111702.

[23] Y. S. Muzychka, E. J. Walsh, P. Walsh, Heat transfer enhancement using laminar gas-liquid segmented plug flows, Journal of Heat Transfer 133 (2011) 041902.

[24] D. Lakehal, G. Larrignon, C. Narayanan, Computational heat transfer and two-phase flow topology in miniature tubes, Microfluidics and Nanofluidics 4 (2008) 261-271.

[25] C. Narayanan, D. Lakehal, Two-phase convective heat transfer in miniature pipes under normal and microgravity conditions, Journal of Heat Transfer 130 (2008) 074502.

[26] P. Urbant, A. Leshansky, Y. Halupovich, On the forced convective heat transport in a droplet-laden flow in microchannels, Microfluidics and Nanofluidics 4 (2008) 533-542.

[27] K. Fukagata, N. Kasagi, P. Ua-arayaporn, T. Himeno, Numerical simulation of gas-liquid two-phase flow and convective heat transfer in a micro tube, International Journal of Heat and Fluid Flow 28 (2007) $72-82$.

[28] V. Talimi, Y. S. Muzychka, S. Kocabiyik, Numerical simulation of the pressure drop and heat transfer of two phase slug flows in microtubes using moving frame of reference technique, International Journal of Heat and Mass Transfer 55 (2012) 6463-6472. 
[29] K. Choi, A. H. C. Ng, R. Fobel, A. R. Wheeler, Digital microfluidics, Annual Review of Analytical Chemistry 5 (2012) 413-440.

[30] M. J. Jebrail, M. S. Bartsch, K. D. Patel, Digital microfluidics: a versatile tool for applications in chemistry, biology and medicine, Lab on a Chip 12 (2012) 2452-2463.

[31] E. Baird, K. Mohseni, Digitized heat transfer: A new paradigm for thermal management of compact micro systems, IEEE Transactions on Components and Packaging Technologies 31 (2008) 143-151.

[32] K. Mohseni, E. S. Baird, Digitized heat transfer using electrowetting on dielectric, Nanoscale and Microscale Thermophysical Engineering 11 (2007) 99-108.

[33] T. M. Squires, S. R. Quake, Microfluidics: Fluid physics at the nanoliter scale, Reviews of Modern Physics 77 (2005) 977-1026.

[34] P. Tabeling, A brief introduction to slippage, droplets and mixing in microfluidic systems, Lab on a Chip 9 (2009) 2428-2436.

[35] Z. Che, N.-T. Nguyen, T. N. Wong, Analysis of chaotic mixing in plugs moving in meandering microchannels, Physical Review E 84 (2011) 066309.

[36] P. Aussillous, D. Quéré, Quick deposition of a fluid on the wall of a tube, Physics of Fluids 12 (2000) 2367-2371.

[37] S. V. Patankar, Numerical Heat Transfer and Fluid Flow, Series in 
computational methods in mechanics and thermal sciences, Hemisphere Pub. Corp., Washington, 1980. 